\title{
Using of Bernstein spectral Galerkin method for solving of weakly singular Volterra-Fredholm integral equations
}

\author{
Majid Erfanian ${ }^{1}$ (D) Hamed Zeidabadi ${ }^{2}$
}

Received: 12 July 2017 / Accepted: 15 March 2018/Published online: 22 March 2018

(C) The Author(s) 2018

\begin{abstract}
In this work, we applied a new method for solving the linear weakly singular mixed Volterra-Fredholm integral equations. We now begin the theoretical study with acquirement of the variational form; in addition, we are using Bernstein spectral Galerkin method to be approximate to my problems. We estimate the error of the method by proved some theorems. Moreover, in the final section, we solved some numerical examples.
\end{abstract}

Keywords Linear weakly singular Volterra-Fredholm integral equation · Finite-element method · Error estimation

Mathematics Subject Classification 45D05 - 65L60

\section{Introduction}

One of the best subjects in the numerical analysis is a finite-element method (FEM). We used (FEM) to solve problems in mathematical physics, integral equations, and engineering, such as electromagnetic potential, fluid flow, radiation heats transfer, laminar boundary-layer theory and mass transport, Abel integral equations, and problem of mechanics or physics [3-5, 7, 18, 20]. For approximating of singular or weakly singular integral equations, there are several numerical method's existences. For example, Baratella and Orsi [8] were introduced weakly singular for Volterra, and discussed on operational matrix method with block-pulse functions by Babolian and Salimi [6]. Furthermore, some author works to be approximate to Abel integral equations, for example, Garza in [12] and Hall in [13] used the wavelet method, Legendre wavelets approximation by Yousefi in [19], Gauss-Jacobi quadrature

Majid Erfanian

erfaniyan@uoz.ac.ir

Hamed Zeidabadi

h.zeidabadi@yahoo.com

1 Department of Science, School of Mathematical Sciences, University of Zabol, Zabol, Iran

2 Faculty of Engineering, Sabzevar University of New Technology, Sabzevar, Iran rule by Fettis in [10], and Piessens and Verbaeten in $[16,17]$ with Chebyshev polynomials of the first kind.

In this paper, we use (FEM) and Bernstein polynomials to acquire an approximate solution for linear weakly singular mixed Volterra-Fredholm integral equation as follows:

$$
\begin{aligned}
& u(x)=f(x)+\int_{a}^{x} \frac{W_{1}(x, t)}{(x-t)^{\alpha}} u(t) \mathrm{d} t+\int_{a}^{b} \frac{W_{2}(x, t)}{(x-t)^{\beta}} u(t) \mathrm{d} t, \\
& 0<\alpha, \beta<1
\end{aligned}
$$

that $f(x), W_{1}(x, t)$, and $W_{2}(x, t)$ are known continuous functions, and $u(x)$ is the unknown function.

\section{Bernstein polynomials and their properties}

On the interval $[a, b]$, the $m+1$ Bernstein basis polynomials (BPs) of degree $m$ are defined as [1]

$$
B_{i, m}(t)=\left(\begin{array}{c}
m \\
i
\end{array}\right) \frac{(t-a)^{i}(b-t)^{m-i}}{(b-a)^{m}}, \quad i=0,1, \ldots, m
$$

In addition, for $i<0$ or $i>m$, we have

$$
B_{i, m}(t)=0 \text {. }
$$

The ten first Bernstein basis polynomials are 
Fig. 1 Bernstein basis polynomials of degrees 1,2 , and 3
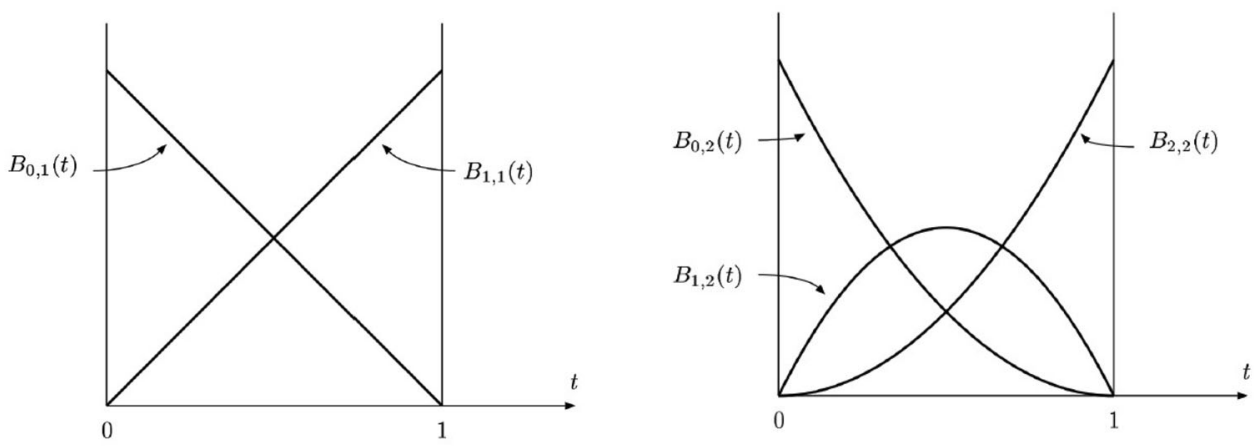

$B_{0,0}(t)=1$,

$B_{0,1}(t)=(1-t), \quad B_{1,1}(t)=t$,

$B_{0,2}(t)=(1-t)^{2}, \quad B_{1,2}(t)=2 t(1-t), \quad B_{2,2}(t)=t^{2}$,

$B_{0,3}(t)=(1-t)^{3}, \quad B_{1,3}(t)=3 t(1-t)^{2}, \quad B_{2,3}(t)=3 t^{2}(1-t), \quad B_{3,3}(t)=t^{3}$.

Therefore, we can be plotted this ten first Bernstein basis polynomials on the unit square as follows (Fig. 1):

\section{Implementation of the Bernstein-Galerkin method}

In this section with using of Galerkin method, we find an approximate solution for Eq. (1). For this purpose, we obtain weak and variational form.

If $\Omega=[a, b] \subset \mathbb{R}$ is an infinite dimensional space, and $\mathbb{V}=H^{0}(\Omega)=L_{2}(\Omega)$.

We let $B: \mathbb{V} \times \mathbb{V} \rightarrow \mathbb{R}$ is a bilinear form and $L: \mathbb{V} \rightarrow \mathbb{R}$ is a linear functional; then for all arbitrary functions $v(x) \in \mathbb{V}$, we have $B(u, v)=L(v)$, where

$$
\begin{aligned}
B(u, v)= & \int_{\Omega} u(x) v(x) \mathrm{d} x-\int_{\Omega} v(x)\left(\int_{a}^{x} \frac{W_{1}(x, t) u(t)}{(x-t)^{\alpha}} \mathrm{d} t\right) \mathrm{d} x \\
& -\int_{\Omega} v(x)\left(\int_{a}^{b} \frac{W_{2}(x, t) u(t)}{(x-t)^{\beta}} \mathrm{d} t\right) \mathrm{d} x,
\end{aligned}
$$

and

$$
L(v)=\int_{\Omega} f(x) v(x) \mathrm{d} x
$$

thus

$$
\begin{aligned}
B\left(\lambda_{1} u+\lambda_{2} w, v\right)= & \int_{\Omega}\left(\lambda_{1} u(x)+\lambda_{2} w(x)\right) v(x) \mathrm{d} x \\
& -\int_{\Omega} v(x) \int_{a}^{x} \frac{W_{1}(x, t)\left(\lambda_{1} u(t)+\lambda_{2} w(t)\right)}{(x-t)^{\alpha}} \mathrm{d} t \mathrm{~d} x \\
& -\int_{\Omega} v(x) \int_{a}^{b} \frac{W_{2}(x, t)\left(\lambda_{1} u(t)+\lambda_{2} w(t)\right)}{(x-t)^{\beta}} \mathrm{d} t \mathrm{~d} x,
\end{aligned}
$$

then, $B$ is a bilinear form:

$B\left(\lambda_{1} u+\lambda_{2} w, v\right)=\lambda_{1} B(u, v)+\lambda_{2} B(w, v)$.

We consider

$u_{h}(x)=\sum_{i=1}^{n} a_{i} \phi_{i}(x), \quad v_{h}(x)=\phi_{j}(x), \quad u_{h}(x), v_{h}(x) \in \mathbb{V}_{h}$

that $V_{h}=\operatorname{span}\left\{\phi_{1}, \phi_{2}, \ldots, \phi_{n}\right\}$ is a subspace of $\mathbb{V}$, and $\left\{\phi_{i}\right\}_{i=1}^{n}$ are a set of Bernstein polynomial functions of degree at most $m$ in each subinterval. Hence, by substituting (4) in variational formulation, we have 


$$
\begin{aligned}
\sum_{i=1}^{n} & a_{i}\left\{\int_{\Omega} \phi_{i}(x) \phi_{j}(x) \mathrm{d} x\right. \\
& -\int_{\Omega} \phi_{j}(x)\left(\int_{0}^{x} \frac{W_{1}(x, t)}{(x-t)^{\alpha}} \phi_{i}(t) \mathrm{d} t\right) \mathrm{d} x \\
& \left.-\int_{\Omega} \phi_{j}(x)\left(\int_{a}^{b} \frac{W_{2}(x, t)}{(x-t)^{\beta}} \phi_{i}(t) \mathrm{d} t\right) \mathrm{d} x\right\} \\
& -\int_{\Omega} g(x) \phi_{j}(x) \mathrm{d} x=0 .
\end{aligned}
$$

Now, for $i, j=1,2, \ldots, n$, we define

$$
\begin{aligned}
C_{i, j}= & \int_{\Omega} \phi_{i}(x) \phi_{j}(x) \mathrm{d} x \\
& -\int_{\Omega} \phi_{j}(x) \int_{0}^{x} \frac{W_{1}(x, t)}{(x-t)^{\alpha}} \phi_{i}(t) \mathrm{d} t \mathrm{~d} x \\
& -\int_{\Omega} \phi_{j}(x) \int_{a}^{b} \frac{W_{2}(x, t)}{(x-t)^{\beta}} \phi_{i}(t) \mathrm{d} t \mathrm{~d} x,
\end{aligned}
$$

and

$G_{j}=\int_{\Omega} g(x) \phi_{j}(x) \mathrm{d} x, \quad j=1,2, \ldots, n$

thus

$\sum_{i=1}^{n} C_{i j} a_{i}=G_{j}, \quad j=1,2, \ldots, n$.

From system (8), we have

$$
C^{T} \mathbf{A}=\mathbf{G}
$$

that

$$
\begin{aligned}
& \mathbf{A}=\left[a_{1}, a_{2}, \ldots, a_{n}\right]^{T}, \quad \mathbf{G}=\left[G_{1}, G_{2}, \ldots, G_{n}\right]^{T}, \\
& \quad C=\left[C_{i j}\right], \quad i, j=1,2, \ldots, n .
\end{aligned}
$$

By solving of the system (9), we can obtain approximate solution of Eq. (1).

\section{Error analysis}

In this section, using the theorem, we get an upper bound for the error of our method, and we proved that the order of convergence is a $O\left(h^{\zeta}\right)$. For this purpose, suppose that $\mathbb{V}$ and $B$ are a Hilbert space and symmetric, respectively.

Definition 1 If $B$ is a $\mathbb{V}$-elliptic bilinear form, then an inner product energy is a $(.,):. \mathbb{V} \times \mathbb{V} \rightarrow \mathbb{R}$ and the energy norm as

$\|u\|_{E}^{2}=(u, u)_{B}=B(u, u)$.

Definition 2 For operator $\Pi: \mathbb{V} \rightarrow \mathbb{V}_{h}$, projection operators as
$\Pi u=\tilde{u}_{h}=\sum_{i=1}^{n} \tilde{a}_{i} \phi_{i}(x)$.

Theorem 1 Let $\alpha>0$, then bilinear form $B$, defined by (3) is a $\mathbb{V}$-ellipticity and Eq. (1) has a unique solution, and order of convergence is a $O\left(h^{\zeta}\right)$.

Proof From Eq. (3), we have

$$
\begin{aligned}
|B(u, v)|= & \mid \int_{\Omega} u(x) v(x) \mathrm{d} x-\int_{\Omega} v(x) \int_{a}^{x} \frac{W_{1}(x, t) u(t)}{(x-t)^{\alpha}} \mathrm{d} t \mathrm{~d} x \\
& -\int_{\Omega} v(x) \int_{a}^{b} \frac{W_{2}(x, t) u(t)}{(x-t)^{\beta}} \mathrm{d} t \mathrm{~d} x \mid
\end{aligned}
$$

with using of the Cauchy-Schwarz inequality and $L_{2}$-norm, we have

$$
\begin{aligned}
& |B(u, v)| \leq\|u\|_{L_{2}(\Omega)}\|v\|_{L_{2}(\Omega)}+W_{1}\left|\int_{a}^{b} v(x) \int_{a}^{x} \frac{u(t)}{(x-t)^{\alpha}} \mathrm{d} t \mathrm{~d} x\right| \\
& +W_{2}\left|\int_{a}^{b} v(x) \int_{a}^{b} \frac{u(t)}{(x-t)^{\beta}} \mathrm{d} t \mathrm{~d} x\right| \\
& =\|u\|_{L_{2}(\Omega)}\|v\|_{L_{2}(\Omega)} \\
& +W_{1}\left|\int_{a}^{b} v(x) u\left(\eta_{x}\right) \int_{a}^{x} \frac{1}{(x-t)^{\alpha}} \mathrm{d} t \mathrm{~d} x\right| \\
& +W_{2}\left|\int_{a}^{b} v(x) u\left(\zeta_{x}\right) \int_{a}^{b} \frac{1}{(x-t)^{\beta}} \mathrm{d} t \mathrm{~d} x\right| \\
& \leq\|u\|_{L_{2}(\Omega)}\|v\|_{L_{2}(\Omega)}+W_{1} \\
& \left|\int_{a}^{b} v(x) u\left(\eta_{x}\right) \frac{1}{1-\alpha}(x-t)^{1-\alpha}\right|_{t=a}^{t=x} \mathrm{~d} x \mid \\
& +W_{2}\left|\int_{a}^{b} v(x) u\left(\zeta_{x}\right) \frac{1}{1-\beta}(x-t)^{1-\beta}\right|_{t=a}^{t=b} \mathrm{~d} x \mid \\
& \leq\|u\|_{L_{2}(\Omega)}\|v\|_{L_{2}(\Omega)} \\
& +\frac{W_{1}(b-a)^{1-\alpha}}{1-\alpha}\left|\int_{a}^{b} v(x) u\left(\eta_{x}\right) \mathrm{d} x\right| \\
& +\frac{W_{2}(b-a)^{1-\beta}}{1-\beta}\left|\int_{a}^{b} v(x) u\left(\zeta_{x}\right) \mathrm{d} x\right| \\
& \leq\left(1+\frac{W_{1}(b-a)^{1-\alpha}}{1-\alpha}+\frac{W_{2}(b-a)^{1-\beta}}{1-\beta}\right)\|u\|_{L^{2}(\Omega)}\|v\|_{L^{2}(\Omega)},
\end{aligned}
$$

where

$$
\begin{aligned}
& W_{1}=\max \left|W_{1}(x, t)\right|, \quad x \in[a, b], \quad \text { and } \quad t \in[a, x], \\
& W_{2}=\max \left|W_{2}(x, t)\right|, \quad x \in[a, b], \quad \text { and } t \in[a, b] \text {, }
\end{aligned}
$$

then $B$ is a continuous. Furthermore, we proved $V$-ellipticity of $B$; for this purpose, we have 


$$
\begin{aligned}
B(v, v)= & \int_{\Omega} v(x) v(x) \mathrm{d} x-\int_{\Omega} v(x) \int_{a}^{x} \frac{W_{1}(x, t) v(t)}{(x-t)^{\alpha}} \mathrm{d} t \mathrm{~d} x \\
& -\int_{\Omega} v(x) \int_{a}^{b} \frac{W_{2}(x, t) v(t)}{(x-t)^{\beta}} \mathrm{d} t \mathrm{~d} x \\
\geq & \|v\|_{L_{2}(\Omega)}^{2}-W_{1}\left(\frac{(b-a)^{1-\alpha}}{1-\alpha}\right)\|v\|_{L_{2}}^{2} \\
& -W_{2}\left(\frac{(b-a)^{1-\beta}}{1-\beta}\right)\|v\|_{L_{2}}^{2}=(\eta)\|v\|_{L_{2}(\Omega)}^{2},
\end{aligned}
$$

then

$$
B(v, v) \geq(\eta)\|v\|_{L_{2}(\Omega)}^{2},
$$

where

$\eta=1-W_{1}\left(\frac{(b-a)^{1-\alpha}}{1-\alpha}\right)-W_{2}\left(\frac{(b-a)^{1-\beta}}{1-\beta}\right)$

thus $B$ is a $V$-ellipticity; therefore, using of Lax-Milgram theorem and $V$-ellipticity of $B$, Eq. (1) has a unique solution. Suppose $u_{h}$ is an approximate solution, so we have

$B\left(u, v_{h}\right)=L\left(v_{h}\right), \quad \forall v_{h} \in V_{h}$,

and

$B\left(u_{h}, v_{h}\right)=L\left(v_{h}\right), \quad \forall v_{h} \in V_{h}$.

If $e=u-u_{h}$ that $u$ are an exact solution of Eq. (1), then

$B\left(e, v_{h}\right)=0, \quad \forall v_{h} \in V_{h}$.

By Schwartz's inequality, and relation between energy norm and inner product, we have

$|B(v, w)| \leq\|v\|_{E}\|w\|_{E}, \quad \forall v, w \in V$.

Using (15), we have

$\left(e, v_{h}\right)_{B}=B\left(e, v_{h}\right)=0$.

Therefore, $e$ is an orthogonal for any $v_{h}$. Using

$\left\|u-u_{h}\right\|_{E}=\min \left\{\left\|u-v_{h}\right\|_{E} ; v_{h} \in V_{h}\right\}$,

and Cea's Lemma [9], for each particular $\tilde{v}_{h}$ in $V_{h}$, we have $\left\|u-v_{h}\right\|_{V} \leq \frac{M}{\eta} \inf \left\|u-v_{h}\right\|_{V}, \quad v_{h} \in V_{v}$

where

$M=\left(1+\frac{W_{1}(b-a)^{1-\alpha}}{1-\alpha}+\frac{W_{2}(b-a)^{1-\beta}}{1-\beta}\right)$.

Since

$\inf \left\|u-v_{h}\right\|_{V} \leq\left\|u-\tilde{v}_{h}\right\|_{V}$

if $\tilde{v}_{h}$ is equal to $\tilde{u}_{h}$, then
$\left\|u-u_{h}\right\|_{V} \leq \frac{M}{\eta}\left\|u-\tilde{u}_{h}\right\|_{V}$.

If we get an upper bounded for the interpolation error, we have

$\left\|u-\tilde{u}_{h}\right\|_{V} \leq c h^{\zeta}, \quad \zeta>0$,

that $c$ is not dependent of $h$; therefore

$\left\|u-u_{h}\right\|_{V} \leq \frac{c M}{\eta} h^{\zeta}$

Thus, $h \rightarrow 0$, and the order of convergence is a $O\left(h^{\zeta}\right)$,

\section{Numerical examples}

Example 1 Consider the linear weakly singular VolterraFredholm integral equation:

$$
\begin{aligned}
& u(x)-\int_{0}^{x} \frac{1}{(x-t)^{\frac{1}{4}}} u(t) \mathrm{d} t-\int_{0}^{1} \frac{1}{(x-t)^{\frac{1}{4}}} u(t) \mathrm{d} t=f(x), \\
& 0<x \leq 1
\end{aligned}
$$

that

$$
\begin{aligned}
f(x)= & \frac{1}{1155}\left(-512 x^{3}-256 x^{2}+144 x+112\right)(x-1)^{\frac{3}{4}} \\
& -x^{3}+x^{2}-\frac{256}{231} x^{\frac{11}{4}}+\frac{1024}{1155} x^{\frac{15}{4}},
\end{aligned}
$$

and the exact solution is a $u(x)=x^{2}(1-x)$.

With using Bernstein basis polynomials of degree 2, and $M=5$, the results of obtained are presented in Table 1 and Fig. 2.

Example 2 In this example, we consider

$$
\begin{aligned}
& u(x)-\int_{0}^{x} \frac{1}{(x-t)^{\frac{1}{3}}} u(t) \mathrm{d} t-\int_{0}^{1} \frac{1}{(x-t)^{\frac{1}{2}}} u(t) \mathrm{d} t=f(x), \\
& 0<x \leq 1
\end{aligned}
$$

that

$f(x)=x-\frac{9}{10} x^{\frac{5}{3}}-\frac{4}{3} x^{\frac{3}{2}}+\frac{2}{3} \sqrt{x-1}+\frac{4}{3} x \sqrt{x-1}$.

In addition, the exact solution is $u(x)=x$.

With using of Bernstein basis polynomials of degree 2, and $M=5$, the results of obtained are presented in Table 2 and Fig. 3.

Example 3 Consider the equation: 
Table 1 Numerical results for Example 1

Fig. 2 Diagrams of exact and numerical solutions and graph of error for Example 1
Table 2 Numerical results for Example 2

\begin{tabular}{lllll}
\hline$t$ & Exact solution & Present method & Finite-element method & RBF method \\
\hline 0.1 & 0.0009000 & 0.0091050 & 0.007818 & 0.016214 \\
0.2 & 0.0320000 & 0.0318483 & 0.031339 & 0.039239 \\
0.3 & 0.0630000 & 0.0630459 & 0.062834 & 0.069327 \\
0.4 & 0.0960000 & 0.0959182 & 0.096335 & 0.100756 \\
0.5 & 0.1250000 & 0.1250346 & 0.125836 & 0.127732 \\
0.6 & 0.1440000 & 0.1439448 & 0.145334 & 0.144997 \\
0.7 & 0.1470000 & 0.1470188 & 0.148841 & 0.147017 \\
0.8 & 0.1280000 & 0.1280360 & 0.130317 & 0.127756 \\
0.9 & 0.8100000 & 0.0809419 & 0.083896 & 0.082547 \\
1 & 0.0000000 & 0.0005053 & 0.003101 & 0.010865 \\
\hline
\end{tabular}
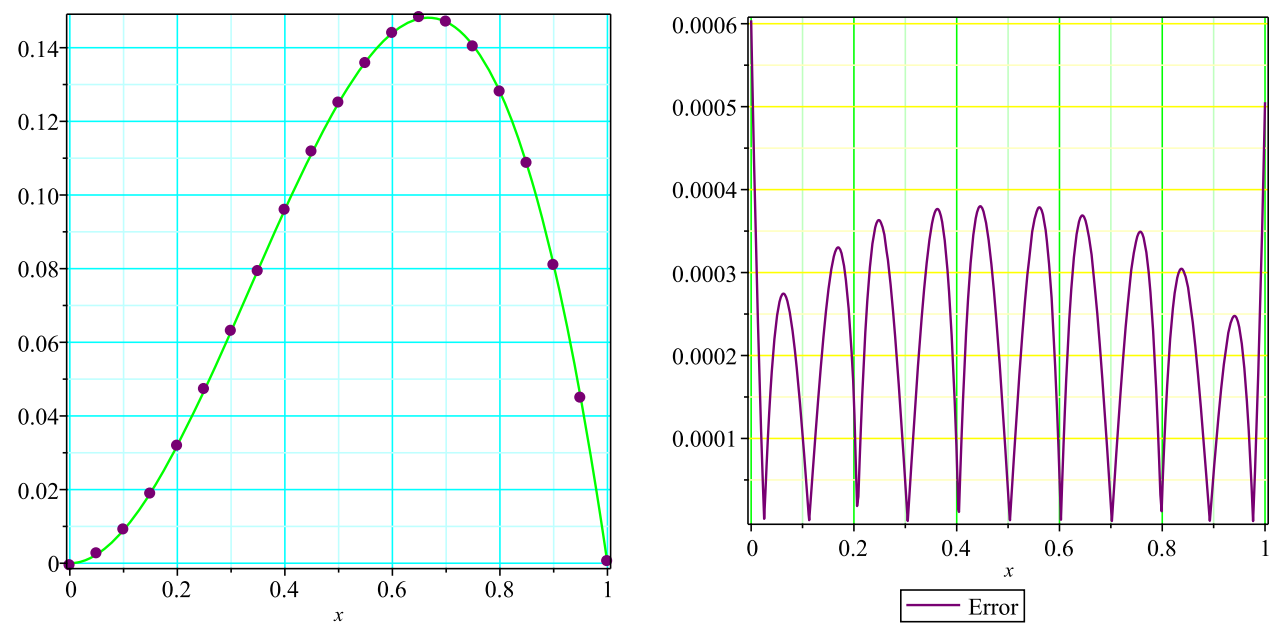

\begin{tabular}{lllll}
\hline$t$ & Exact solution & Present method & Finite-element method & RBF method \\
\hline 0.1 & 0.1000000 & 0.0999999 & 0.0999997 & 0.0999999 \\
0.2 & 0.2000000 & 0.2000000 & 0.1999999 & 0.1999999 \\
0.3 & 0.3000000 & 0.3000000 & 0.2999999 & 0.2999999 \\
0.4 & 0.4000000 & 0.4000000 & 0.3999999 & 0.3999998 \\
0.5 & 0.5000000 & 0.5000000 & 0.4999999 & 0.4999995 \\
0.6 & 0.6000000 & 0.6000000 & 0.5999999 & 0.5999991 \\
0.7 & 0.7000000 & 0.7000000 & 0.6999999 & 0.6999983 \\
0.8 & 0.8000000 & 0.7999999 & 0.7999999 & 0.7999964 \\
0.9 & 0.9000000 & 0.9000000 & 0.9000000 & 0.8999921 \\
1 & 1.0000000 & 1.0000000 & 0.9999998 & 0.9999835 \\
\hline
\end{tabular}

With using of Bernstein basis polynomials of degree 2, and $M=5$, the results of obtained are presented in Table 3 and Fig. 4.

\section{Conclusions}

$f(x)=\exp (x)(\operatorname{erf}(\sqrt{x-1}) \sqrt{\pi}-2 \operatorname{erf}(\sqrt{x}) \sqrt{\pi}+1)$,

and the exact solution is $u(x)=\exp (x)$.
In this paper, we used of Galerkin method and Bernstein polynomials to solving one of the most important linear weakly singular Volterra-Fredholm integral equation, with 
Fig. 3 Diagrams of exact and numerical solutions and graph of error for Example 2

Table 3 Numerical results for Example 3
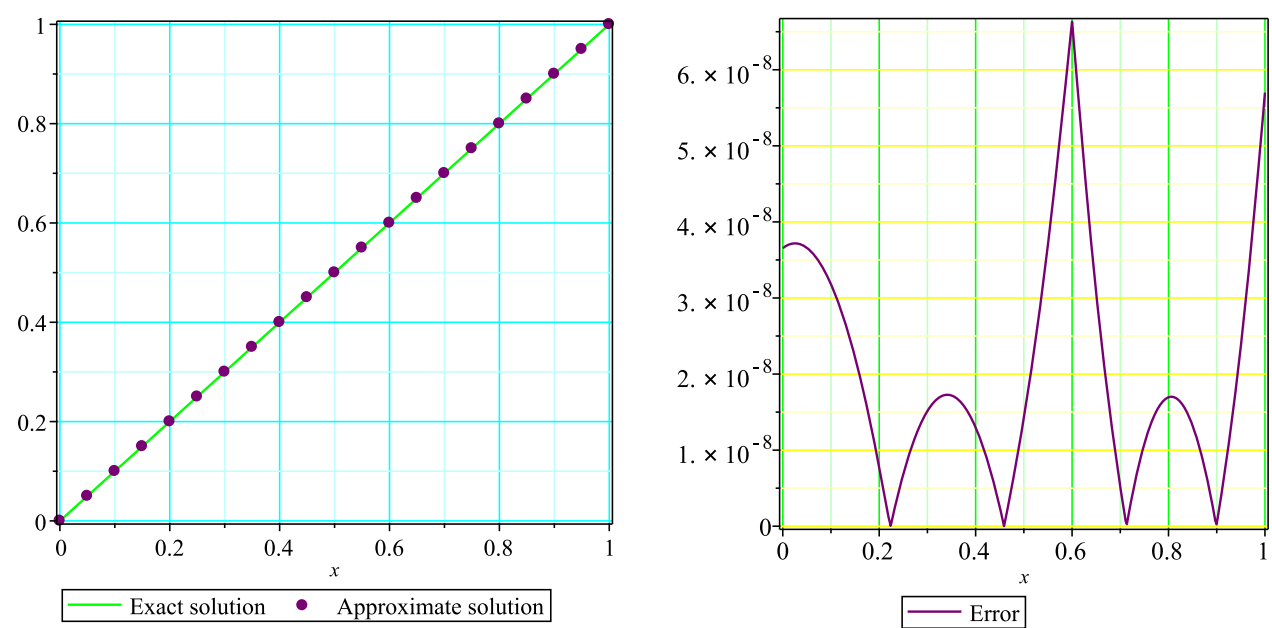

\begin{tabular}{lllll}
\hline$t$ & Exact solution & Present method & Finite-element method & RBF method \\
\hline 0.1 & 1.1051709 & 1.1051108 & 1.1042527 & 1.1051602 \\
0.2 & 1.2214027 & 1.2215436 & 1.2203756 & 1.2214104 \\
0.3 & 1.3498588 & 1.3497954 & 1.3487213 & 1.3498715 \\
0.4 & 1.4918246 & 1.4919761 & 1.4905784 & 1.4918240 \\
0.5 & 1.6487212 & 1.6486511 & 1.6473242 & 1.6487057 \\
0.6 & 1.8221188 & 1.8222743 & 1.8206197 & 1.8221042 \\
0.7 & 2.0137527 & 2.0136890 & 2.0120126 & 2.0137483 \\
0.8 & 2.2255409 & 2.2256379 & 2.2237996 & 2.2254962 \\
0.9 & 2.4596031 & 2.4595848 & 2.4573309 & 2.4593229 \\
1 & 2.7182818 & 2.7181290 & 2.7164838 & 2.7173060 \\
\hline
\end{tabular}

Fig. 4 Diagrams of exact and numerical solutions and graph of error for Example 3
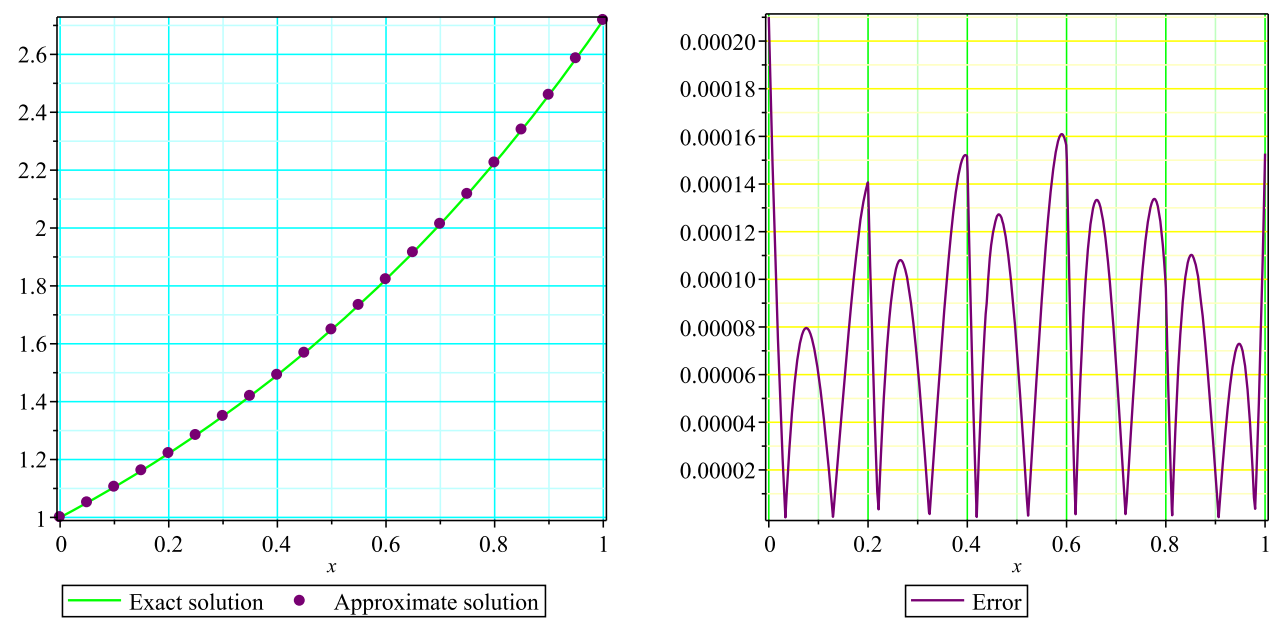

using of Bernstein basis polynomials. We now begin the theoretical study with acquire of the variational form of Eq. (1), and with using of the system (9), we can obtain approximate solution. In section error analysis, we proved that $B$ is a $\mathbb{V}$-ellipticity and Eq. (1) has a unique solution, and order of convergence is a $O\left(h^{\zeta}\right)$. In section Numerical
Examples, we have solved three problems considered, the results obtained are presented in Tables 1,2, and 3, and Figs. 2, 3, and 4, the comparison of results confirms the better accuracy with this method. 
Open Access This article is distributed under the terms of the Creative Commons Attribution 4.0 International License (http://creative commons.org/licenses/by/4.0/), which permits unrestricted use, distribution, and reproduction in any medium, provided you give appropriate credit to the original author(s) and the source, provide a link to the Creative Commons license, and indicate if changes were made.

\section{References}

1. Kreyszig, E.: Introduction Functional Analysis with Applications. Wiley, New York (1978)

2. Alipour, M., Rostamy, D., Baleanu, D.: Solving multi-dimensional FOCPs with inequality constraint by BPs operational matrices. J. Vib. Control 19(16), 2523-2540 (2012)

3. Sparrow, E.M.: Application of variational method to radiation heat-transfer calculations. J. Heat Transfer 82, 375-380 (1960)

4. Ames, W.F.: Nonlinear Partial Differential Equations in Engineering. Academic Press, New York (1965)

5. Atkinson, K.E.: The Numerical Solution of Integral Equations of the Second Kind, Cambridge Monographs Applied and Computational Mathematics (2009)

6. Babolian, E., Salimi Shamloo, A.: Numerical solution of Volterra integral and integro-differential equations of convolution type by using operational matrices of piecewise constant orthogonal functions. J. Comput. Appl. Math. 214(2), 495-508 (2008)

7. Baker, C.T.H.: The Numerical Treatment of Integral Equations. Clarendon Press, Oxford (1977)

8. Baratella, P., Orsi, A.P.: A new approach to the numerical solution of weakly singular Volterra integral equations. J. Comput. Appl. Math. 163, 401-418 (2004)
9. Brenner, S., Ridgway Scott, L.: The Mathematical Theory of Finite Element Methods. Texts in Applied Mathematics. Springer, New York (2007)

10. Fettis, M.E.: On numerical solution of equations of the Abel type. Math. Comput. 18, 491-496 (1964)

11. Galperin, A., Kansa, E.J.: Application of global optimazation and radial basis functions to numerical solution of weakly singular Volterra equations. J. Comput. Appl. Math. 43, 491-499 (2002)

12. Garza, J., Hall, P., Ruymagaart, F.H.: A new method of solving noisy Abel-type equations. J. Math. Anal. Appl. 257, 403-419 (2001)

13. Hall, P., Paige, R., Ruymagaart, F.H.: Using wavelet methods to solve noisy Abel-type equations with discontinuous inputs. J. Multivariate Anal. 86, 72-96 (2003)

14. Huang, L., Huang, Y., Li, X.: Approximate solution of Abel integral equation. J. Comput. Appl. Math. 56, 1748-1757 (2008)

15. Linz, P.: Analytical and Numerical Methods for Volterra Equations. SIAM, Philadelphia (1985)

16. Piessens, R.: Computing integral transforms and solving integral equations using Chebyshev polynomial approximations. J. Comput. Appl. Math. 121, 113-124 (2000)

17. Piessens, R., Verbaeten, P.: Numerical solution of the Abel integral equation. BIT 13, 451-457 (1973)

18. Wazwaz, A.M.: A First Course in Integral Equations. World Scientific Publishing Company, New Jersey (1997)

19. Yousefi, S.A.: Numerical solution of Abel s integral equation by using Legendre wavelets. Appl. Math. Comput. 175, 574-580 (2006)

20. Siekmann, J.: The laminar boundary layer along a flat plate. Z. Flugwiss. 10, 278-281 (1962) 